4. Englund A, Freeman TP, Murray RM, McGuire P. Can we make cannabis safer? Lancet Psychiatry. 2017;4:643-8.

5. National Academies of Sciences, Engineering, Medicine. The Health Effects of Cannabis and Cannabinoids: The Current State of Evidence and Recommendations for Research, January 2017. http://nationalacademies.org/hmd/Reports/2017/ health-effects-of-cannabis-and-cannabinoids.aspx; http://nationalacademies.org/ hmd/ /media/Files/Report\%20Files/2017/Cannabis-Health-Effects/Cannabischapter-highlights.pdf.

6. Morgan CJA, Curran HV. Effects of cannabidiol on schizophrenia-like symptoms in people who use cannabis. Br J Psychiatry. 2008;192:306-7.
7. Schubart CD, Sommer IE, van Gastel WA, Goetgebuer RL, Kahn RS, Boks MP (2011). Cannabis with high cannabidiol content is associated with fewer psychotic experiences. Schizophr Res. 2011;130:216-21.

8. Madras BK, Bergman J, Kohut S, George S, Hurd Y, Lin Z. THC of marijuana upregulates dcc mRNA expression in prefrontal cortex but THC combined with Cannabidiol (CBD) does not: relevance to psychiatric symptoms associated with long term marijuana use? American College of Neuropsychopharmacology 56th Annual Meeting. Palm Springs, CA, 3-7 Dec 2016: Poster T234; 2017.

\title{
Sex differences in schizophrenia: estrogen and mitochondria
}

\author{
Vanessa F. Gonçalves ${ }^{1}$, Ari B. Cuperfain ${ }^{1}$ and James L. Kennedy ${ }^{1,2}$ \\ Neuropsychopharmacology (2019) 44:216-217; https://doi.org/10.1038/s41386-018-0228-0
}

Schizophrenia (SCZ) is a neuropsychiatric disorder which displays sex differences in its clinical manifestations, including women presenting with later age at onset, less severe course, and better response to antipsychotics in comparison with men. Estrogen is a steroid hormone which, in addition to its role in females' reproduction and sex characteristics, contributes to neuroprotection within the central nervous system.

In this context, there is compelling evidence that estrogen has a protective effect in females against the development of neuropsychiatric disorders, particularly SCZ. Estrogen is reported to influence several features of SCZ pathophysiology and to promote the observed sex-difference in the clinical outcomes between males and females. The molecular mechanisms by which estrogen affects SCZ are still largely unknown, but there is evidence that estrogen plays a role in synaptic plasticity, neurogenesis, and neurotransmission, as well as in modulating reactive oxygen species (ROS) burden in the brain [1]. Notably, each of these neurobiological mechanisms is disturbed in the brain of SCZ patients.

More recently, it has been reported that estrogen also modulates mitochondrial function [2]. Mitochondria are the main source of energy for most cellular activities. In the brain, mitochondria are also implicated in many neuronal processes reported to be involved in SCZ pathophysiology, and mitochondrial dysfunction is emerging as a risk factor for the disorder [3]. Over 1000 mitochondrial genes are encoded by the nuclear genome, and also, mitochondria contain their own small genome (mtDNA), which is exclusively maternally inherited. Interestingly, studies have revealed a stronger maternal inheritance of SCZ compared to paternal [4].

Mitochondria have sex-specific features, exhibiting distinct effects in males versus females, and these effects have strong links to neuroprotection. Briefly, studies in a variety of animal models have shown that females' mitochondria have increased biogenesis and oxidative capacity, and greater antioxidant defense with (consequently) reduced generation of ROS, thus less release of mitochondrial apoptotic factors [5]. There is evidence that estrogen might be a key player in this mitochondrial sexual dimorphism. Several nuclear-encoded mitochondrial genes show specific DNA sequences that are targets of estrogen, called estrogen response elements (ERE). The strongest evidence is the finding that estrogen receptor beta binds to estrogen EREs in $m t D N A$, directly regulating its gene transcription. Moreover, with respect to direct biochemical connection for the role of estrogen in mitochondrial function, treatment of cells with estrogen protects against electron transport chain inhibitors [2]. However, the actions of estrogen on mitochondrial functioning that may underlie this hormone's sex-specific effects on brain development and behavior in schizophrenia remain uninvestigated, and are an important target of research in animal models for the disease.

Overall, it appears that estrogen plays a role in SCZ pathophysiology and in protecting females against a more severe course of the disease, probably (but not exclusively) through its regulation of the mitochondrial system. A better understanding of the influence of estrogen on mitochondrial function in the context of SCZ will reveal new insights regarding the pathophysiology of the disease in males versus females. This new perspective will generate novel targets for drug discovery with potential to improve many of the SCZ clinical outcomes, as well as developing future preventive and therapeutic strategies.

\section{ACKNOWLEDGMENTS}

We would like to acknowledge the Judy and Larry Tanenbaum Family Foundation for their support to this study.

\section{FUNDING}

J.L.K. has been funded by Genome Canada, The Ontario Ministry of Research and Innovation, CIHR, a BBRF/NARSAD Distinguished Investigator Award, and the Judy and Larry Tanenbaum Foundation. J.L.K. has also received speaker honoraria and expenses from Eli Lilly, Novartis, and Shire, and consultant honoraria and expenses from Roche, and is a nonpaid member of the SAB of Assurex Health Inc.Competing interests: The authors declare no competing interest.

Publisher's note: Springer Nature remains neutral with regard to jurisdictional claims in published maps and institutional affiliations. 


\section{REFERENCES}

1. Gogos A, Sbisa AM, Sun J, Gibbons A, Udawela M, Dean B. A role for estrogen in schizophrenia: clinical and preclinical findings. Int J Endocrinol. 2015;2015:615356.

2. Liao TL, Tzeng CR, Yu CL, Wang YP, Kao SH. Estrogen receptor beta in mitochondria: implications for mitochondrial bioenergetics and tumorigenesis. Ann N Y Acad Sci. 2015;1350:52-60.
3. Cuperfain AB, Zhang ZL, Kennedy JL, Gonçalves VF. The complex interaction of mitochondrial genetics and mitochondrial pathways in psychiatric disease. Mol Neuropsychiatry. 2018;4(1):52-69.

4. Verge B, Alonso Y, Valero J, Miralles C, Vilella E, Martorell L. Mitochondrial DNA (mtDNA) and schizophrenia. Eur Psychiatry. 2011;26(1):45-56.

5. Ventura-Clapier R, Moulin M, Piquereau J, Lemaire C, Mericskay M, Veksler V, et al. Mitochondria: a central target for sex differences in pathologies. Clin Sci. 2017;131 (9):803-22.

\title{
Vaccines to treat opioid use disorders and to reduce opioid overdoses
}

\author{
Carly Baehr ${ }^{1,2}$ and Marco Pravetoni ${ }^{2,3}$ \\ Neuropsychopharmacology (2019) 44:217-218; https://doi.org/10.1038/s41386-018-0197-3
}

As the incidence of opioid-related fatal overdoses continues to rise in the United States, new therapeutic strategies for opioid use disorders (OUD) are needed. One proposed solution is active immunization with anti-opioid conjugate vaccines, which selectively reduce the effects of their target opioid through production of drug-specific antibodies. As opposed to pharmacotherapies (e.g., methadone, buprenorphine, and naltrexone) targeting opioid receptors in the brain, opioid-specific antibodies operate through a pharmacokinetic mechanism by sequestering the target opioid in serum and reducing its distribution to the brain. Anti-opioid vaccines could provide safe and cost-effective interventions that offer several advantages over current small molecule medications: long-lasting protection that reduces the burden of compliance; no abuse liability or risk of diversion; and due to their selectivity, vaccines do not interfere with endogenous opioids, or with nontarget opioids prescribed for pain management or for treatment of OUD. To improve clinical outcome, vaccines could be used in combination with other medications for OUD.

Extensive preclinical studies have identified a series of lead vaccines selectively targeting heroin, oxycodone, hydrocodone, or fentanyl (e.g., [1-3]). Anti-opioid vaccines effectively reduce distribution of the target opioid to the brain, and reduce opioidinduced behavior, including drug self-administration, in mice, rats, or nonhuman primates. Notably, vaccine efficacy in reducing opioid distribution to the brain depends on the target opioid, its dose, and route of exposure [2], highlighting the need to consider variables such as patterns of drug use in study design of clinical trials. Supporting a role for vaccines in overdose prevention, vaccination reduces opioid-induced respiratory depression and bradycardia, two significant factors in overdose-related fatalities [4]. Additionally, anti-opioid vaccines do not interfere with naloxone reversal of opioid toxicity [4] and improve survival following a lethal heroin dose [5].

Clinical evaluation of first-generation nicotine and cocaine vaccines has shown that only a subset of immunized subjects produced levels of drug-specific antibodies sufficient for efficacy. Therefore, it is critical to optimize vaccine formulations to maximize efficacy, to understand the immunological mechanisms underlying effective immune responses, and to identify biomarkers predictive of individual variability. Multiple studies have focused on vaccine design, including optimization of hapten and linker chemistry, choice of carrier protein and adjuvant, and development of novel carriers and delivery platforms. Effective formulations of vaccines against heroin have included the TLR9 agonist CpG [5] and liposomes containing the TLR4 agonist monophosphoryl lipid A [3]. The efficacy of an oxycodone vaccine was enhanced by shifting IgG subclass distribution through inhibition of interleukin-4 signaling, both indicating a pharmacological target for vaccine development, and granting insight into mechanisms underlying vaccine efficacy [6]. Additionally, the pre-immunization frequency of hapten-specific B cell population subsets correlated with vaccine efficacy, suggesting that subjects likely to generate clinically effective opioid-specific antibody responses could be identified prior to vaccination [7]. Accelerating the translation of vaccines for OUD will benefit from rational design of more effective vaccine components, development of clinically viable formulations, and biomarkers supporting patient stratification.

Mounting preclinical data provide proof of selectivity and efficacy for anti-opioid vaccines, demonstrating their potential to treat OUD and reduce incidence of opioid overdoses. Testing these vaccines in clinical trials is warranted.

\section{ACKNOWLEDGEMENTS}

This work is supported by a Hennepin Healthcare Research Institute award (M.P.), and by the NIH under grants U01 DA038876 (M.P.), R01 DA041730 (M.P.), and T32DA007097 (C.B.).Competing interests: M.P. is the inventor of "Cytokine Signaling Immunomodulators and Methods," International Application No. PCT/US2017/031907 filed on May 10, 2017. C.B. declares no competing interests.

Publisher's note: Springer Nature remains neutral with regard to jurisdictional claims in published maps and institutional affiliations.

\footnotetext{
${ }^{1}$ Department of Veterinary Population Medicine, University of Minnesota, St. Paul, MN, USA; ${ }^{2}$ Hennepin Healthcare Research Institute (formerly Minneapolis Medical Research Foundation), Minneapolis, MN, USA and ${ }^{3}$ Departments of Medicine and Pharmacology, Center for Immunology, University of Minnesota, Minneapolis, MN, USA

Correspondence: Marco Pravetoni (prave001@umn.edu)
}

Published online: 12 September 2018 